\title{
Zeolite membranes for chemical separation
}

Petroleum is not just a fuel, it is a complex mixture of chemical species. This is why it is such a rich source of many essentia chemicals for pharmaceutical and plastics manufacturing. However, the distillation differs for separating these incredibly energy intensive. Dr Motomu Sakai at the Waseda University Nano Life Innovatio Research Organization is developing an alternative approach to distillation for chemical separation. His zeolt membranes are capable of separating several of the most with excellent selectivity.

Dof hydrocarbon-based chemicats. As well as its value for refining into fuels, a significant proportion of the world's petroleum use is for the refinement and separation of chen species for chemical feedstocks. for further choms can then be used manufacture high-value products such as plastics, pharmaceuticals and other industrial chemicals.

Separation of petroleum into these different chemical species involves process called distillation. In distillation, the petroleum is heated to over 600 degrees until it starts to boil. As it boils, vapour is released up a long column that is held at a temperature gradient. The bottom of the column close to the boiling petroleum is hottest and the top is the coolest part. As the vapour rises, it starts to cool, and when it reaches a region of the equal to the boiling po ent of som of the chemicals in the vapor they will begin to condense into liquid and run off into a collection C. tray. The distillation petroleum are the same processes used whisky and tequila.

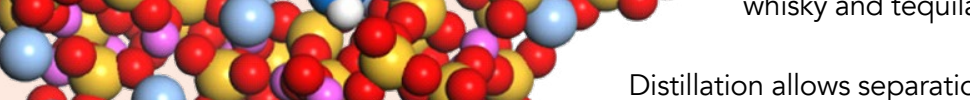
H. 00 Distillation allows separation -. Cocer as as boiling point that is related to the structural arrangement of its ato with larger compounds typically having higher boiling points. While the separation power of distillation is good, keeping the petroleum at high
temperatures for sufficient time to allow

Dr Sakai utilises the alkene-binding power
of silver in his custom silver-exchanged the evaporation, condensation and collection of the desired compound is incredibly energy intensive.

This is why Dr Motomu Sakai at the Waseda University Nano Life Innovation Research Organization is proposing of chemicals in petroleum. Rather than trying to make distllation more energy efficient he is pushing for a different approach using zeolite membranes to isolate the chemicals of interest. Using his expertise to create custom zeolites for chemical separation in this way has the potential to offer huge energy savings while remaining a selective method with good efficiency.

\section{ZEOLITE TRAPS}

Zeolites are a broad class of materials that are made from aluminium and slicon and have a very special structure. The arrangement of the atoms in the zeolite is such that small spaces, pockets rin ming them porous. In these small, small ionic species within their strupture, making them highly efficient adsorbents and catalysts. The long channel-like networks of pockets mean that gaseous or liquid samples can be passed throug them where they act as type of sieve to filter out any chemicals of interest. This trapping power means that zeolites are often used in water treatment, as they can help to remove small molecules that the taste or safety of water.

While zeolites do occur naturally, they are not particularly common and so most zeolites are made synthetically. the synthesis process, he properties a thericans ean be tallored for specific can be changed to trap bigger

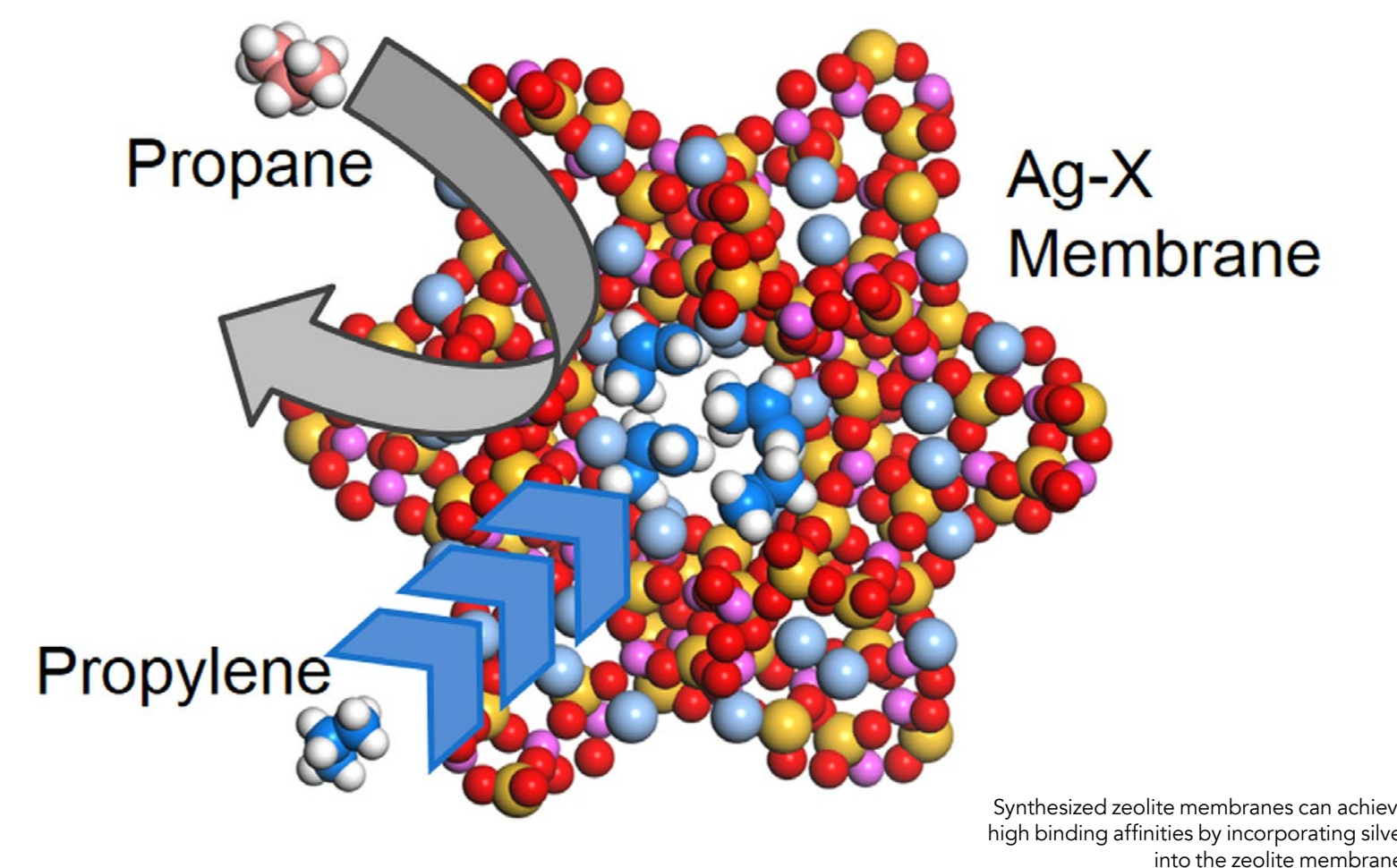

smaller molecules. In Dr Sakai's case, alongside his collaborator Professor Matsukata, he has tailored the chemical composition and structure of his zeolites to create high precision chemical sieves that trap only the chemical species they have been designed to target.

\section{FILTERING WALL}

Membranes are a type of selective

barrier that only allow certain species

to pass. Most of our cells are surrounded

by protective membrane walls that

coll As weave as keeping the cell intact, the mentl

acts a chemical sieve so the concentrates of ions in the cell remain carefully balanced and these membranes play a key role in maintaining cellular health and function.

Dr Sakai aims use his zeolites as part of a chemically selective membrane, but rather than doing this for cells, he is interested in applying the idea to petrochemical refinement. Mimicking the very high selectivity of cells for specific chemicals with the zeolite membranes is very important as the extracted chemicals from petrochemi feedstocks must be extremely pure Even in manufactuing of polym Enemicals can cause issues with the polymerisation process and negatively impact the properties of the final plastics of materials made.

Synthetic polymer membranes have already been shown to be capable of separating different alkenes from each other. Alkenes (hydrocarbons tha

incorporating silver into the polymer membrane, which has a strong affinity in alkenes. This is how these membran are able to achieve excellent selectivity

ZEOLTT MEMBRANES Dr Sakai has been utilising the

Has the potential to offer huge energy savings while remaining a selective method with good efficiency.

contain a strong carbon-carbon double bond) are one of the most widely used chemical feedstocks, particularly for plastics manufacture. These polymer membranes have been able to achieve high binding affinities for alkenes by

alkene-binding power of silver in his custom silver-exchanged zeolite nembranes. These are zeolites With silver incorporated into their alkenes. For separation of propane and
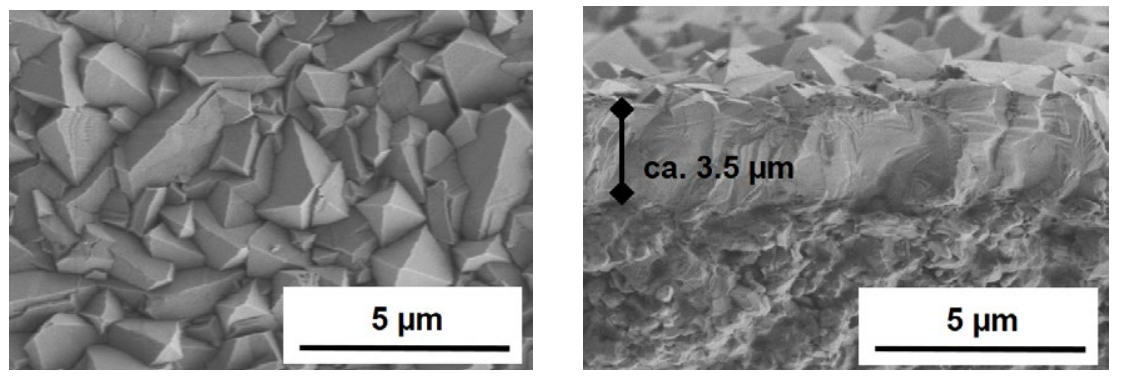

FE-SEM membrane surface. 


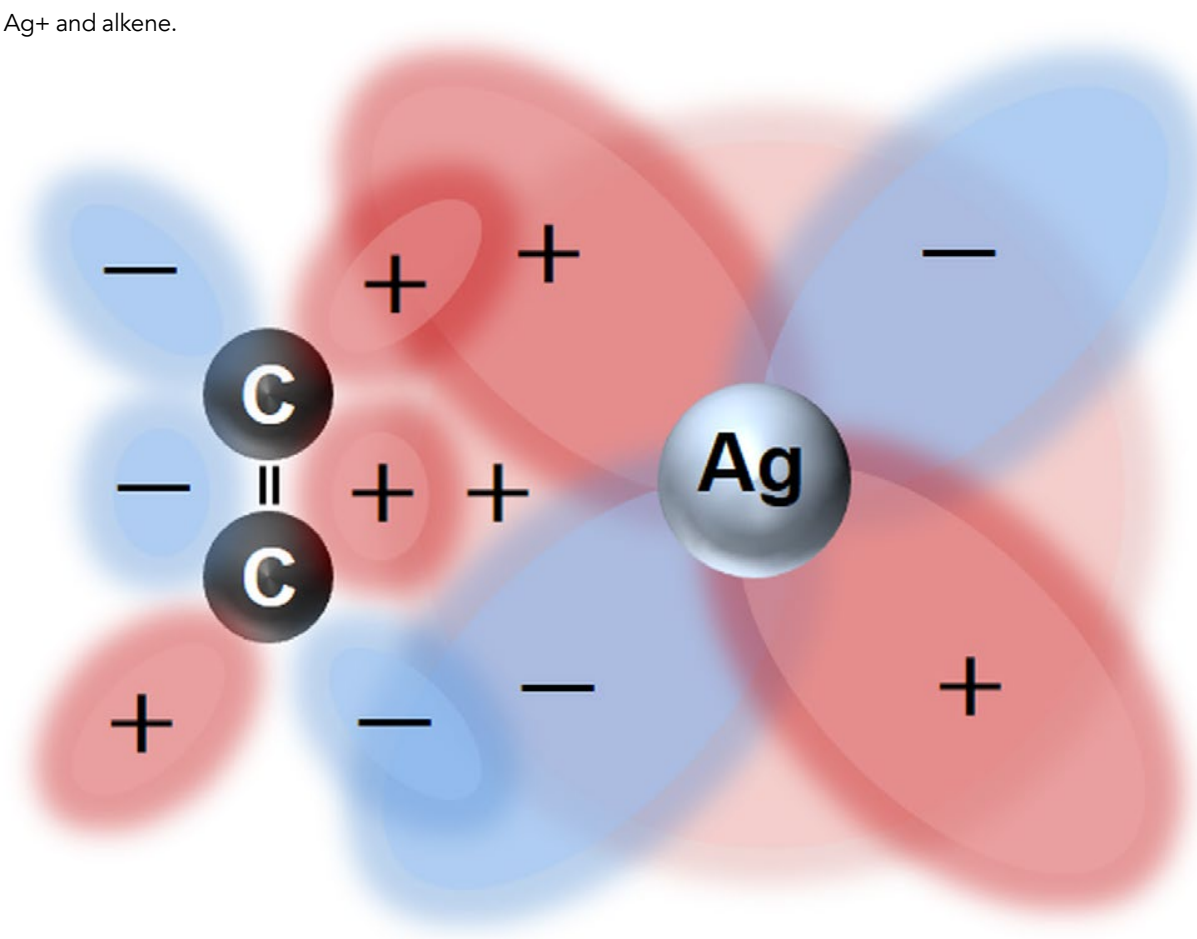

\section{Behind the Research}

\section{Dr Motomu Sakai}

E: saka.moto@aoni.waseda.jp T: +81-3-5286-3850 W: http://www.matsukata-lab.jp/

\section{Research Objectives}

Dr Sakai's research is focused on advancing sustainable and environmental technologies.

\section{Detail}

169-8555

-4-1 Okubo, Shinjuku-ku

Tokyo, Japan

Bio

otomu Sakai is a junior researcher and instructor at the Research Organization of Nano \& Life Innovation, Waseda University, Japan. His research is focused on the preparation of zeolite membranes for separation and analysis of separation mechanisms. He aims to reduce consumption energy for separation in petroleum and petrochemical industries through the use of zeolite membranes. Dr Sakai's zeolites showed a very high the mixture passes through the zeolite, the propylene is bound preferentially and blocks penetration of the remaining propane. Following the affinity, only the attached propene can pass throug the membrane and then be used as a high-purity chemical feedstock. As well s developing useful zeolite membranes, Dr Sakairs work is also providing achieve their high separation efficiencies. high separation efficiencies mean that the use of these membranes shows The zeolite membranes have also_great promise as a way of replacing shown similar promising results for the distillation for extraction of feedstock chemicals and simulations also

\section{Dr Sakail's work is also providing} important insights into the mechanisms through which these zeolite species achieve their high separation efficiencies.

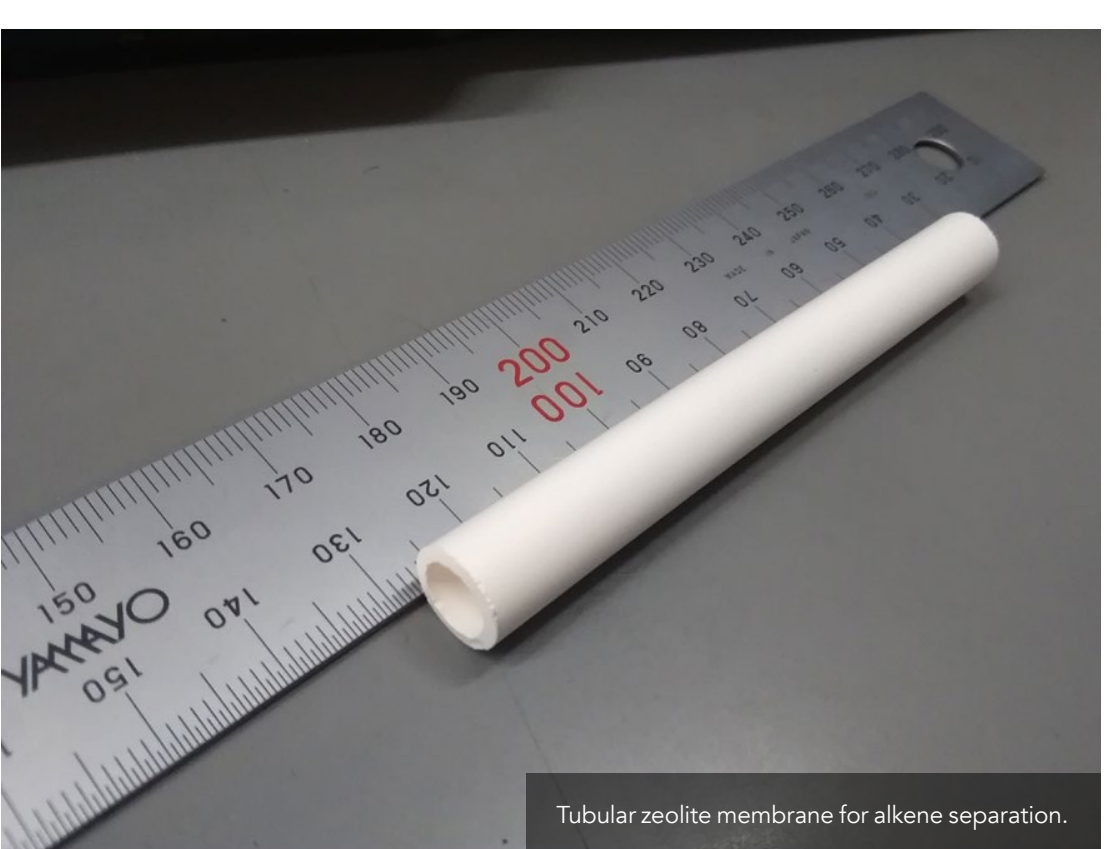

performed in the group suggest that the levels of separation achievable through the use of such zeolites but comparable to distillation, and energy cost.

Through the careful design and study of zeolite membranes, Dr Sakai's separation will help to reduce the huge energy expenditure associated with chemical refinement. Not only that but the chemically robust nature of zeolite membranes means they are likely to have long working lifetimes for the separation process and be resistan to attack or degradation by other chemical species, so will not need frequent replacement.
Funding

ST CREST (Japan Science and Technology agency, Create REvolutionary technological seeds for Science and Technology innovation program), Grant Number PMJCR1324, Japan.

\section{Collaborators}

shimo (Co-author)
(Co-autho)

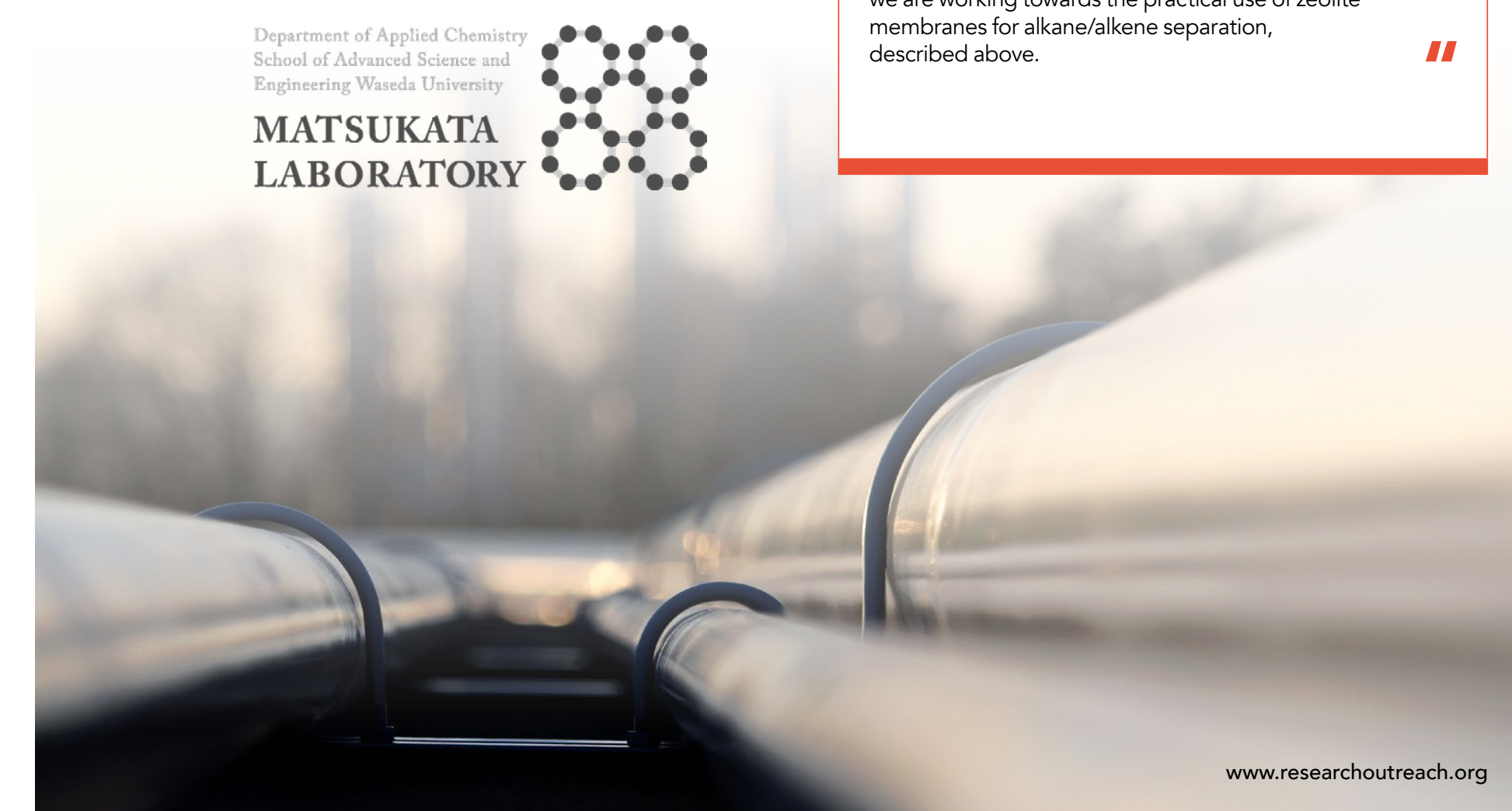

References

Sakai, M., Sasaki, Y., Tomono, T., Seshimo, M. and Matsukata, M., (2019), ACS Applied Materials and Interfaces, 11, 4145-4151. DOI: 10.1021/acsami.8b2015.

Sakai, M., Fujimaki, N., Kobayashi, G., Yasuda, N., Oshima Y., Seshimo, M., Matsukata, M., (2019), Microporous and Mesoporous Materials, 284, 360-365, 10.1016/].

\section{Personal Response}

\section{Which hydrocarbons will your target next with you} zeolite membranes?

II Now we are strenuously aiming for $p$-xylene separation from xylene isomers, and n-alkane separation from light oil. There is a large demand for $p$-xylene as a raw material for PET bottles. The current process of p-xylene production o- and $m$-xylenes. We have developed a zeolite membran that allows $p$-xylene to preferentially permeate.

n-Alkane is used for fuels, heat storage materials, and as a lubricant. Because our new zeolite membrane has high up to $500^{\circ} \mathrm{C}$. We believe that this membrane can be used in the membrane-distillation hybrid process and contribute to reduction of energy consumption in petroleum refinery. At the same time as developing novel zeolite membranes, we are working towards the practical use of zeolite 